\title{
Complete Monotonicity of the Representative Consumer's Discount Factor
}

\author{
Chiaki Hara* \\ Institute of Economic Research, Kyoto University
}

August 25, 2007

\begin{abstract}
A univariate real-valued function is said to be completely monotone if it takes positive values and alternate the signs of its higher order derivatives, starting from everywhere negative first derivatives. We prove that the representative consumer's discount factor of a continuous-time economy under uncertainty is a power function of some completely monotone function of time satisfying certain boundary conditions if and only if it may be derived from a group of consumers having constant and equal relative risk aversion, and constant and yet possibly unequal discount rates.
\end{abstract}

JEL Classification Codes: D51, D53, D61, D81, D91, E43, G12.

Keywords: Complete monotonicity, discount factor, discount rate, representative consumer, expected utility, time additivity, relative risk aversion, Bernstein's theorem.

\section{Introduction}

In this paper, we are concerned with a continuous-time economy under uncertainty populated by multiple consumers. We assume that all consumers have time-additive utility functions and constant subjective time discount rates, although the rates may differ across them. We are interested in how the heterogeneity in discount rates affects the term structure of interest rates.

As is often done in the analysis of asset pricing and efficient risk sharing, we will consider the problem of maximizing the weighted sum of individual consumers' utilities subject to the resource feasibility constraint. The value function of this constrained maximization problem, as a function of aggregate consumption processes, is called the representative consumer's utility

\footnotetext{
${ }^{*}$ My first gratitude goes to Chenghu Ma, who gave me extremely helpful comments as the discussant when I presented this paper at the fourth Workshop on General Equilibrium Theory in Asia (GETA 2007), held at the National University of Singapore on August 2007. Atsushi Kajii gave me useful suggestions on the exposition of the paper. I am also grateful to workshop participants at Hitotsubashi University (Tokyo, Japan), Kyoto University (Kyoto, Japan), and GETA 2007. The Grant in Aid for Specially Promoted Research from Japan Society for the Promotion of Sciences on "Economic Analysis on Intergenerational Problems" and the grant from Ishii Memorial Foundation for the Promotion of Financial Studies on "Microeconomic Foundations of the Term Structure of Interest Rates" are gratefully acknowledged. My email address is hara@kier.kyoto-u.ac.jp.
} 
function. Since the individual consumers' utility functions are time-additive, so is the representative consumer's. Let us call the integrand of a consumer's utility function his felicity function. Our study is focused on how the heterogeneity in the individual consumers' discount rates affects the representative consumer's felicity function.

Gollier and Zeckhauser (2005, Proposition 5) showed that if all individual consumers' felicity functions exhibit decreasing absolute risk aversion, then the representative consumer's discount rates are decreasing with respect to time. They, moreover, showed that if there are infinitely many consumers, having some appropriately chosen utility weights, and their (constant) discount rates are exponentially distributed, and if their felicity functions exhibit constant and equal relative risk aversion (a special case of decreasing absolute aversion), then the representative consumer's discount rate is a hyperbolic function of time (a special case of decreasing discount rates). These results show that the representative consumer's time preferences may well be, both qualitatively and quantitatively, quite different from individual consumers' counterparts when their subjective discount rates are heterogeneous. In particular, even if they all have constant discount rates, the representative consumer would typically not. This fact has significant implication on asset pricing and the term structure of interest rates. It also casts some doubt on the applicability of the standard representative-consumer model where the representative consumer has a time-additive utility function with a constant discount rate.

In this paper, we develop the analysis of Gollier and Zeckhauser (2005) further by maintaining the assumption that all consumers have constant and equal relative risk aversion but dispensing with the assumption that the heterogeneous discount rates are exponentially distributed. Then the representative consumer would not necessarily exhibit hyperbolic discounting, and, yet, we can fully characterize the representative consumer's discount factor. We shall do this by proposing a sub-class, denoted by $\mathscr{H}$, of completely monotone functions. An infinitely many times differentiable function $h: \boldsymbol{R}_{++} \rightarrow \boldsymbol{R}$ is said to be completely monotone if $(-1)^{n} h^{(n)}(t) \geq 0$ for every non-negative integer $n$, where $h^{(n)}$ is the $n$-th derivative of $h$ if $n \geq 1$ and $h^{(0)}=h$ by convention. We will impose additional restrictions on the asymptotic behavior of $h(t)$ as $t \rightarrow \infty$ and $t \rightarrow 0$ to define the sub-class $\mathscr{H}$, and show that the representative consumer's discount factor is a power function of some function in $\mathscr{H}$ (which is a function of time but not of aggregate consumption levels) if and only if it can be derived from a group of consumers having constant and equal constant relative risk aversion, and constant but not necessarily equal discount rates. This result indicates what aspects of, say, the term structure of interest rates can or cannot be accounted for by the heterogeneity of consumers' discount rates in models of constant and equal relative risk aversion.

In general, the representative consumer's utility function is subordinated to the choice of utility weights. While Gollier and Zeckhauser (2005) found utility weights for the representative consumer to exhibit hyperbolic discounting when the individual consumers' (constant) discount rates are exponentially distributed, they did not check whether the profile of such utility weights are the unique one or there may be other utility weights that give rise to the same discount factor for the representative consumer. In this paper, we show that the representative consumer's 
discount factor uniquely determines the profile of individual consumers' utility weights. This is true not only when the individual consumers' discount rates are exponentially distributed (and hence the representative consumer exhibits hyperbolic discounting) but also when they are arbitrarily distributed (and hence we only know that his discount factor is a power function of some function in $\mathscr{H}$ ). Since his discount factor can be uniquely determined by the term structure of interest rates and the utility weights are monotonically related with the wealth shares, this results also tells us that once we observe the term structure of interest rates, we can fully recover the wealth distribution across consumers of various (constant) discount rates. It also allows us, in principle, to assess the plausibility of the tem structure models by comparing the implied wealth distribution with the empirically observed one.

The mathematical fact behind these results are the celebrated theorem by S. Bernstein, which asserts that a function is completely monotone if and only if it can be represented as an integral of negative exponential functions, where the integral is taken over constants multiplied to the arguments of the negative exponential functions. It is easy to check from the first-order conditions of a solution to the constrained maximization problem of the weighted sum of utilities that if all consumers have constant and equal relative risk aversion, then the representative consumer's discount factor is a power function of some (completely monotone) function in $\mathscr{H}$. To establish the converse, we need, for any given (completely monotone) function $\mathscr{H}$, to specify the individual consumers' utility weights. This is the step where we need Bernstein's theorem.

This paper is organized as follows. Section 2 lays out the setting of the paper and gives some preliminary results. Section 3 reviews known results on completely monotone functions and proposes the sub-class $\mathscr{H}$. Section 4 presents the main result of this paper. Section 5 concludes.

\section{Setup and Preliminary Results}

The economy is subject to uncertainty, which is represented by a probability measure space $(\Omega, \mathscr{F}, P)$. The time span is $\boldsymbol{R}_{+}=[0, \infty)$, which is of continuous time and infinite length, although it could be $[0, T]$ with $0<T<\infty$, which is of finite length. The gradual information revelation is represented by a filtration $\left(\mathscr{F}_{t}\right)_{t \in \boldsymbol{R}_{+}}$. There is only one type of good on each time and state. ${ }^{1}$

We allow the number of consumers present in the economy to be finite and infinite. Formally, we let $(A, \mathscr{A}, \nu)$ be a finite measure space of (names of) consumers. If $A$ is a finite set, $\mathscr{A}$ is the power set of $A$, and $\nu$ is the counting measure on $A$, then the consumption sector consists of finitely many consumers. If, on the other hand, $A$ is the unit interval $[0,1], \mathscr{A}$ is the Borel $\sigma$-field $\mathscr{B}([0,1])$, and $\nu$ is (the restriction of) the Lebesgue measure on $\mathscr{B}([0,1])$, then the consumption sector consists of infinitely many consumers, each of whom is negligible in size relative to the

\footnotetext{
${ }^{1}$ Just as the analysis of Gollier and Zeckhauser (2005), the subsequent analysis would still be valid even in the absence of uncertainty. Although we could simplify our model by restricting our attention to a deterministic economy, we have chosen to incorporate uncertainty to make our model immediately applicable to asset pricing theory.
} 
total population of the economy. For each $B \in \mathscr{A}, \nu(B)$ is the proportion of the consumers belonging to $B$ in the entire consumption sector.

We assume that the consumers have time-additive utility functions over consumption processes, which exhibit constant and equal relative risk aversion, and constant but possibly unequal discount rates. Formally, let $\beta>0$ and $u: \boldsymbol{R}_{++} \rightarrow \boldsymbol{R}$ satisfy $u^{\prime}(x)=x^{-\beta}$ for every $x \in \boldsymbol{R}_{++}$. Let $\rho: A \rightarrow \boldsymbol{R}_{++}$be measurable, where $\boldsymbol{R}_{++}$is endowed with the Borel $\sigma$-field $\mathscr{B}\left(\boldsymbol{R}_{++}\right)$. Then the utility function $U_{a}$ of consumer $a$ over consumption processes is defined by

$$
U_{a}\left(c^{a}\right)=E\left(\int_{0}^{\infty} \exp (-\rho(a) t) u\left(c_{t}^{a}\right) d t\right)
$$

where $c^{a}=\left(c_{t}^{a}\right)_{t \in \boldsymbol{R}_{+}} \cdot 2,3$

To find a Pareto efficient allocation of a given aggregate consumption process $c=\left(c_{t}\right)_{t \in \boldsymbol{R}_{+}}$ and its supporting (decentralizing) state-price deflator, it is sufficient to let $\lambda: A \rightarrow \boldsymbol{R}_{++}$be a measurable function and consider the constrained maximization problem

$$
\begin{array}{ll}
\max _{\left(c^{a}\right)_{a \in A}} & \int_{A} \lambda(a) U_{a}\left(c^{a}\right) d \nu(a) \\
\text { subject to } & \int_{A} c^{a} d \nu(a)=c .
\end{array}
$$

The objective function of this constrained maximization problem is additively separable across time and states:

$$
\begin{aligned}
& \int_{A} \lambda(a) U_{a}\left(c^{a}\right) d \nu(a) \\
= & \int_{A} \lambda(a) E\left(\int_{0}^{\infty} \exp (-\rho(a) t) u\left(c_{t}^{a}\right) d t\right) d \nu(a) \\
= & E\left(\int_{0}^{\infty} \int_{A} \lambda(a) \exp (-\rho(a) t) u\left(c_{t}^{a}\right) d \nu(a) d t\right) \\
= & \int_{\Omega \times \boldsymbol{R}_{+}}\left(\int_{A} \lambda(a) \exp (-\rho(a) t) u\left(c_{t}^{a}(\omega)\right) d \nu(a)\right) d(P \otimes \mu)(\omega, t),
\end{aligned}
$$

where $\mu$ is the Lebesgue measure. The constraint, $\int_{A} c^{a} d \nu(a)=c$, is, of course, separable across time and states. To find the solution to the constrained maximization problem, therefore, it suffices to denote by $\mathscr{I}$ the set of all integrable functions defined on $A$ and taking values in

\footnotetext{
${ }^{2}$ This and other integrals in the subsequent analysis need not be well defined without no additional assumptions on $c^{a}$ and other stochastic processes. But the subsequent argument depends only on the first-order conditions of (utility or social welfare) maximization problems, which must necessarily hold whenever there is a solution to the problem under consideration. We shall therefore be implicit about these additional assumptions.

${ }^{3}$ Although the assumption of constant and equal relative risk aversion is quite stringent, there are good reasons to restrict our attention to this case. First, this assumption allows us to compare our results with the result in Gollier and Zeckhauser (2005) on the representative consumer's hyperbolic discounting, as the latter uses the same assumption. Second, under this assumption, if all consumers had the same (constant) discount rate, then the representative consumer would have the same constant relative risk aversion and the same (constant) discount rate as the individual consumers. Hence any anomalies in the representative consumer's discount rate can be attributed to the heterogeneity in the individual consumers' discount rates and nothing else.
} 
$\boldsymbol{R}_{++}$and solve

$$
\begin{array}{ll}
\max _{\iota \in \mathscr{I}} & \int_{A} \lambda(a) \exp (-\rho(a) t) u(\iota(a)) d \nu(a) \\
\text { subject to } & \int_{A} \iota(a) d \nu(a)=x .
\end{array}
$$

for every $(x, t) \in \boldsymbol{R}_{++} \times \boldsymbol{R}_{+}$.

If there is a solution to (1), it must be essentially unique with respect to $\nu$ because $u$ is strictly concave. Assuming its existence, we denote the unique solution by $f(x, t) \in \mathscr{I}$ and write $f_{a}(x, t)$ instead of $f(x, t)(a)$. Then, for every $(x, t)$, by the first-order condition for the solution to (1),

$$
f_{a}(x, t)=\frac{(\lambda(a) \exp (-\rho(a) t))^{1 / \beta}}{\kappa(t)} x
$$

for almost every $a \in A$, where

$$
\kappa(t)=\int_{A}(\lambda(a) \exp (-\rho(a) t))^{1 / \beta} d \nu(a)
$$

This shows that the mutual fund theorem holds at each time $t$ across states, but not across time, because the consumption share $(\kappa(t))^{-1}(\lambda(a) \exp (-\rho(a) t))^{1 / \beta}$ depends on $t$ unless $\rho$ is an essentially constant function of $a$, that is, all consumers' discount rates are equal.

The representative consumer's felicity function $v: \boldsymbol{R}_{++} \times \boldsymbol{R}_{+} \rightarrow \boldsymbol{R}$ is defined as the value function of (1):

$$
v(x, t)=\int_{A} \lambda(a) \exp (-\rho(a) t) u\left(f_{a}(x, t)\right) d \nu(a) .
$$

His utility function over the aggregate consumption processes is defined through time additivity by

$$
U(c)=E\left(\int_{0}^{\infty} v\left(c_{t}, t\right) d t\right) .
$$

It follows from (2) that $v(x, t)=d(t) u(x)$, where $d(t)=(h(t))^{\beta}$ and

$$
h(t)=\int_{A}(\lambda(a))^{1 / \beta} \exp \left(-\frac{\rho(a)}{\beta} t\right) d \nu(a)
$$

for every $t \in \boldsymbol{R}_{+}$. The function $d: \boldsymbol{R}_{+} \rightarrow \boldsymbol{R}$ is the representative consumer's discount factor. Note that in order for $v$ (and, thus, $U$ ) to be well defined, it is necessary that $h(0)<\infty$. This is equivalent to saying that the function $a \mapsto(\lambda(a))^{1 / \beta}$ is integrable with respect to $\nu$.

Define the representative consumer's impatience $r: \boldsymbol{R}_{+} \rightarrow \boldsymbol{R}_{++}$by $r(t)=-d^{\prime}(t) / d(t)$, then

$$
\frac{d(t)}{d(0)}=\exp \left(-\int_{0}^{t} r(\tau) d \tau\right)
$$

for every $t \in \boldsymbol{R}_{+}$. Thus the representative consumer's impatience $r$ is his continuously compounded instantaneous subjective discount rate as a function of time. Unlike the case of individual consumers, this is not constant but varies with $t$ unless all individual consumers have 
the same discount rate. Since $d(t)=(h(t))^{\beta}$,

$$
r(t)=-\frac{h^{\prime}(t)}{h(t)} \beta .
$$

Hence, to identify how the discount rate $r$ depends on time $t$, it is sufficient to identify how $-h^{\prime}(t) / h(t)$ varies with $t$.

The representative consumer is useful to identify asset prices, because his marginal utility process, $\left(\partial v\left(c_{t}, t\right) / \partial x\right)_{t \in \boldsymbol{R}_{+}}$, serves as a state price process. This means that the price at time $t$, relative to the current consumption, of an asset with dividend (rate) process $\delta=\left(\delta_{t}\right)_{t \in \boldsymbol{R}_{+}}$is equal to the discounted sum of its future dividends:

$$
E\left(\int_{t}^{\infty} \frac{\partial v\left(c_{\tau}, \tau\right) / \partial x}{\partial v\left(c_{t}, t\right) / \partial x} \delta_{\tau} d \tau\right)
$$

Moreover, although we analyze the Pareto efficient allocations, if the asset markets are complete, then our analysis is applicable to the equilibrium allocations and asset prices. This is because the first welfare theorem holds in complete markets, so that the equilibrium allocations are Pareto efficient and the equilibrium asset prices are given by the marginal utility process. Since $u$ is concave, the second welfare theorem also holds, so that every Pareto efficient allocation is an equilibrium allocation for some distribution of initial endowments. Hence an analysis of Pareto efficient allocations is nothing but an analysis of equilibrium allocations.

Our findings in this section can be summarized as follows. First, the representative consumer's felicity function $v$ is multiplicatively separable between time $t$ and consumption level $x$. Second, it has constant relative risk aversion $\beta$, just as the individual consumers. Third, the discount rate is not necessarily constant. Moreover, since $\partial v(x, t) / \partial x=(h(t))^{\beta} x^{-\beta}$, by Fubini's theorem,

$$
E\left(\int_{t}^{\infty} \frac{\partial v\left(c_{\tau}, \tau\right) / \partial x}{\partial v\left(c_{t}, t\right) / \partial x} \delta_{\tau} d \tau\right)=\int_{t}^{\infty}\left(\frac{h(\tau)}{h(t)}\right)^{\beta} E\left(\left(\frac{c_{\tau}}{c_{t}}\right)^{-\beta} \delta_{\tau}\right) d \tau
$$

Thus, knowing the properties of function $h$ is useful to identify asset prices. In the rest of this paper, we shall explore such properties by making use of the fact that $h$ is a completely monotone function of $t$.

\section{Complete Monotonicity}

A function $h: \boldsymbol{R}_{++} \rightarrow \boldsymbol{R}$ is completely monotone if it is infinitely many times differentiable and $(-1)^{n} h^{(n)}(t) \geq 0$ for every non-negative integer $n$ and every $t \in \boldsymbol{R}_{+}$, where $h^{(n)}$ is the $n$-th derivative of $h$ if $n \geq 1$ and $h^{(0)}=h$. In this section, we propose a sub-class of completely monotone functions that satisfy some boundary conditions which will later turn out to characterize the representative consumer's discount factor.

We start with presenting a well known characterization of completely monotone functions. Feller (1966, Chapter XIII, Section 4) and Billingsley (1995, Chapter 4, Section 22) are standard references on this topic. 
Theorem 1 (Bernstein) For every completely monotone function $h$, there exist a unique Borel measure $\mu$ on $\boldsymbol{R}_{+}$such that

$$
h(t)=\int_{\boldsymbol{R}_{+}} \exp (-q t) d \mu(q)
$$

for every $t \in \boldsymbol{R}_{++}$. Moreover, $\mu(Q)<\infty$ for every bounded interval $Q \subset \boldsymbol{R}_{+}$.

If $h$ is defined by (8), then by Lebesgue's dominated convergence theorem,

$$
h^{(n)}(t)=\int_{\boldsymbol{R}_{+}}(-q)^{n} \exp (-q t) d \mu(q)
$$

for every $n$ and $t$. This shows that if $\mu$ is concentrated on 0 , then $h$ is a constant function, but otherwise, $(-1)^{n} h^{(n)}(t)>0$ for every non-negative integer $n$ and every $t \in \boldsymbol{R}_{++}$. In this case, we put on record the following result, which will be used to analyze the representative consumer's impatience.

Proposition 1 Define $h: \boldsymbol{R}_{++} \rightarrow \boldsymbol{R}$ by (8) and suppose that $\mu$ is not concentrated on $\{0\}$. Then

$$
\frac{d}{d t}\left(-\frac{h^{(n+1)}(t)}{h^{(n)}(t)}\right) \leq 0
$$

for every non-negative integer $n$ and every $t \in \boldsymbol{R}_{++}$. The weak inequality holds as an equality if and only if $\mu$ is concentrated on a single point of $\boldsymbol{R}_{++}$.

Proof of Proposition 1 Since

$$
\frac{d}{d t}\left(-\frac{h^{(n+1)}(t)}{h^{(n)}(t)}\right)=\frac{1}{\left(h^{(n)}(t)\right)^{2}}\left(\left(h^{(n+1)}(t)\right)^{2}-h^{(n+2)}(t) h^{(n)}(t)\right),
$$

it suffices to show that

$$
h^{(n+2)}(t) h^{(n)}(t) \geq\left(h^{(n+1)}(t)\right)^{2},
$$

and that the weak inequality holds as an equality if and only if $\mu$ is concentrated on a single point of $\boldsymbol{R}_{++}$. To do so, let $\mu_{t}$ be the Borel measure on $\boldsymbol{R}_{+}$of which the Radon-Nikodym derivative with respect to $\mu$ is equal to $\exp (-q t)$. Since

$$
h^{(n)}(t)=(-1)^{n} \int_{\boldsymbol{R}_{+}} q^{n} d \mu_{t}(q),
$$

the $n$-th power function $q^{n}$ is integrable with respect to $\mu_{t}$ for every non-negative integer $n$. 
Thus, by the Cauchy-Schwartz inequality,

$$
\begin{aligned}
h^{(n+2)}(t) h^{(n)}(t) & =\left(\int_{\boldsymbol{R}_{+}}\left(q^{(n+2) / 2}\right)^{2} d \mu_{t}(q)\right)\left(\int_{\boldsymbol{R}_{+}}\left(q^{n / 2}\right)^{2} d \mu_{t}(q)\right) \\
& \geq\left(\int_{\boldsymbol{R}_{+}} q^{(n+2) / 2} q^{n / 2} d \mu_{t}(q)\right)^{2} \\
& =\left(\int_{\boldsymbol{R}_{+}} q^{n+1} d \mu_{t}(q)\right)^{2} \\
& =\left(h^{(n+1)}(t)\right)^{2} .
\end{aligned}
$$

Here the weak inequality holds as an equality if $\mu$, and hence $\mu_{t}$, is degenerated is degenerated on a single point, in which case $h$ is a negative exponential function. Otherwise, it holds as a strict inequality because the two functions $q \mapsto q^{(n+2) / 2}$ and $q \mapsto q^{n / 2}$ are not scalar multiples of each other on any set on which $\mu_{t}$ takes full measure.

The following proposition specifies the class of completely monotone functions that may arise as the representative consumer's discount factor. It is, in essence, stated in Feller (1966, Chapter XIII, Section 4). We omit the proof.

Proposition 2 For every function $h: \boldsymbol{R}_{++} \rightarrow \boldsymbol{R}$, the following two conditions are equivalent.

1. $h$ is completely monotone and satisfies

$$
0=\inf _{t \in \boldsymbol{R}_{++}} h(t) \leq \sup _{t \in \boldsymbol{R}_{++}} h(t)<\infty .
$$

2. There exist a finite Borel measure $\mu$ on $\boldsymbol{R}_{++}$such that

$$
h(t)=\int_{\boldsymbol{R}_{++}} \exp (-q t) d \mu(q)
$$

for every $t \in \boldsymbol{R}_{++}$.

Moreover, then, such a $\mu$ is unique.

Since every completely monotone function is non-increasing, (9) means that $h(t) \rightarrow 0$ as $t \rightarrow \infty$, and $h(t)$ converges to some finite number as $t \rightarrow 0$. We denote the set of all functions satisfying either (and hence both) of these two properties by $\mathscr{H}$. Below is a class of examples of completely monotone functions motivated by the example of Gollier and Zeckhauser (2005, Section IV) on hyperbolic discounting.

Example 1 Let $\alpha, \theta$, and $\kappa$ be non-negative numbers, and define $h$ by

$$
h(t)=\frac{1}{(t+\theta)^{\alpha}}+\kappa
$$


Then $h$ is completely monotone for any choice of $\alpha \geq 0, \theta \geq 0$, and $\kappa \geq 0$, but $h \in \mathscr{H}$ if and only if $\alpha>0, \theta>0$, and $\kappa=0$. It is easy to prove this claim by using Condition 1 of Proposition 2, but in the sequel, we do so by identifying the Borel measure $\mu$ satisfying $h(t)=\int_{\boldsymbol{R}_{+}} \exp (-q t) d \mu(q)$ for every $t \in \boldsymbol{R}_{++}$.

First, if $\alpha=0$, then $h$ is the constant function taking value $1+\kappa$, and if we let $\mu$ be the Borel measure concentrating weight $1+\kappa$ on $\{0\}$, then $h(t)=\int_{\boldsymbol{R}_{+}} \exp (-q t) d \mu(q)$. Since $\mu$ does not satisfy Condition 2 of Proposition 2 and hence $h \notin \mathscr{H}$.

Next, suppose that $\alpha>0$. Then let $\mu_{\text {ac }}$ be the (finite or infinite) Borel measure of which the density function (Radon-Nikodym derivative with respect to the Lebesgue measure) $g: \boldsymbol{R}_{++} \rightarrow$ $\boldsymbol{R}_{+}$is defined by

$$
g(q)=\frac{q^{\alpha-1} \exp (-\theta q)}{\Gamma(\alpha)}
$$

where $\Gamma(\alpha)=\int_{0}^{\infty} s^{\alpha-1} \exp (-s) d s$, that is, $\Gamma$ is the gamma function. Let $\mu_{\mathrm{s}}$ be the Borel measure concentrating weight $\kappa$ on $\{0\}$. Then let $\mu=\mu_{\mathrm{ac}}+\mu_{\mathrm{s}}$. Of course, $\mu=\mu_{\mathrm{ac}}$ if $\kappa=0$. We shall now prove that $h(t)=\int_{\boldsymbol{R}_{+}} \exp (-q t) d \mu(q)$ for every $t \in \boldsymbol{R}_{++}$. Indeed, $\int_{\boldsymbol{R}_{+}} \exp (-q t) d \mu_{\mathrm{s}}(q)=\kappa$ for every $t \in \boldsymbol{R}_{++}$. As for the integral with respect to $\mu_{\mathrm{ac}}$,

$$
\begin{aligned}
& \int_{\boldsymbol{R}_{+}} \exp (-q t) d \mu_{\mathrm{ac}}(q) \\
= & \int_{\boldsymbol{R}_{++}} \exp (-q t) g(q) d q \\
= & \frac{1}{\Gamma(\alpha)} \int_{0}^{\infty} q^{\alpha-1} \exp (-(t+\theta) q) d q
\end{aligned}
$$

By the change of the variable $s=(t+\theta) q$, we see that this is equal to

$$
\begin{aligned}
& \frac{1}{\Gamma(\alpha)} \int_{0}^{\infty}\left(\frac{s}{t+\theta}\right)^{\alpha-1} \exp (-s) \frac{1}{t+\theta} d s \\
= & \frac{1}{\Gamma(\alpha)} \frac{1}{(t+\theta)^{\alpha}} \int_{0}^{\infty} s^{\alpha-1} \exp (-s) d s \\
= & \frac{1}{(t+\theta)^{\alpha}} .
\end{aligned}
$$

Therefore,

$$
\int_{\boldsymbol{R}_{+}} \exp (-q t) d \mu(q)=\int_{\boldsymbol{R}_{+}} \exp (-q t) d \mu_{\mathrm{ac}}(q)+\int_{\boldsymbol{R}_{+}} \exp (-q t) d \mu_{\mathrm{s}}(q)=h(t) .
$$

The measure $\mu$ puts zero weight on $\{0\}$ if and only if $\kappa=0$. It is finite if and only if $g$ is integrable (with respect to the Lebesgue measure), which is, in turn, true if and only if $\theta>0 .{ }^{4}$ Therefore, by Condition 2 of Proposition $2, h \in \mathscr{H}$ if and only if $\alpha>0, \kappa=0$, and $\theta>0$.

The functional form of (11) is closely related to hyperbolic discounting when $\alpha>0$ and $\kappa=0$. Indeed, if $\alpha>0$ and $\kappa=0$, and if the representative consumer's discount factor $d$ is

\footnotetext{
${ }^{4}$ By the same change-of-variable argument as above, we can show that $\int_{\boldsymbol{R}_{+}} g(q) d q=\theta^{-\alpha}$ whenever $\theta>0$.
} 
a power function of $h$ (as in the case of constant and equal relative risk aversion), then $d$ can also be written in the same form as (11), albeit the parameter $\alpha$ replaced by another positive constant. If, nevertheless, we write $d(t)=(t+\theta)^{-\alpha}$ for the simplicity of notation, then

$$
r(t)=-\frac{d^{\prime}(t)}{d(t)}=\frac{\alpha}{t+\theta},
$$

that is, the representative consumer's discount rate $r$ is a hyperbolic function of $t$. Moreover, the converse also holds: if his discount rate $r$ satisfies (12), then the corresponding discount factor $d$ is a positive multiple of some function of the form (11).

\section{Representative Consumer's Discount Factor}

In this section, we fully characterize the representative consumer's discount factor by the subset $\mathscr{H}$ of completely monotone functions. First, we show that for any distribution of the individual consumers' discount rates and utility weight, the representative consumer's discount factor is a power function of some function in $\mathscr{H}$.

Proposition 3 If the function $a \mapsto(\lambda(a))^{1 / \beta}$ is integrable with respect to $\nu$ and if $h: \boldsymbol{R}_{++} \rightarrow \boldsymbol{R}$ is defined by (5), then $h \in \mathscr{H}$.

Proof of Proposition 3 By Proposition 2, it is sufficient to find a finite Borel measure $\mu$ on $\boldsymbol{R}_{++}$for which (8) holds. To do so, denote by $\mathscr{B}$ the sub- $\sigma$-field of $\mathscr{A}$ generated by $\rho$. Since $a \mapsto(\lambda(a))^{1 / \beta}$ is integrable, define a function $\bar{\lambda}: A \rightarrow \boldsymbol{R}_{++}$so that $a \mapsto(\bar{\lambda}(a))^{1 / \beta}$ is (a version of) the conditional expectation of $a \mapsto(\lambda(a))^{1 / \beta}$ given $\mathscr{B}$. Since $a \mapsto \exp (-\rho(a) t / \beta)$ is measurable with respect to $\mathscr{B}$, Theorem 34.3 of Billingsley (1995) implies that

$$
\int_{A}(\lambda(a))^{1 / \beta} \exp \left(-\frac{\rho(a)}{\beta} t\right) d \nu(a)=\int_{A}(\bar{\lambda}(a))^{1 / \beta} \exp \left(-\frac{\rho(a)}{\beta} t\right) d \nu(a) .
$$

We see that the two functions defining utility weights, $\lambda$ and $\bar{\lambda}$, give rise to the same discount factor. By Theorem 20.1 of Billingsley (1995), there exists a measurable function $g: \boldsymbol{R}_{++} \rightarrow$ $\boldsymbol{R}_{++}$such that $\bar{\lambda}(a)=g(\rho(a))$. Then, by (5), (13), and the change-of-variable formula,

$$
h(t)=\int_{\boldsymbol{R}_{++}}(g(s))^{1 / \beta} \exp \left(-\frac{s}{\beta} t\right) d\left(\nu \circ \rho^{-1}\right)(s) .
$$

for every $t \in \boldsymbol{R}_{+}$. By a slight abuse of notation, we also denote by $\beta$ the function of multiplying $\beta$, that is, $\beta(q)=\beta q$ for every $q \in \boldsymbol{R}$. Then, by (14) and, again, the change-of-variable formula,

$$
\begin{aligned}
h(t) & =\int_{\boldsymbol{R}_{++}}(g(s))^{1 / \beta} \exp \left(-\beta^{-1}(s) t\right) d\left(\nu \circ \rho^{-1}\right)(s) \\
& =\int_{\boldsymbol{R}_{++}} \exp (-q t)(g(\beta q))^{1 / \beta} d\left(\left(\nu \circ \rho^{-1}\right) \circ \beta\right)(q) .
\end{aligned}
$$


Denote by $\mu$ the Borel measure on $\boldsymbol{R}_{++}$of which the Radon-Nikodym derivative with respect to $\left(\nu \circ \rho^{-1}\right) \circ \beta$ is the function $q \mapsto(g(\beta q))^{1 / \beta}$. Then (8) holds. Moreover, the function $q \mapsto(g(\beta q))^{1 / \beta}$ is integrable with respect to $\left(\nu \circ \rho^{-1}\right) \circ \beta$ because $\left(\nu \circ \rho^{-1}\right) \circ \beta=\nu \circ\left(\beta^{-1} \circ \rho\right)^{-1}$ and the function $a \mapsto(\bar{\lambda}(a))^{1 / \beta}$ is integrable with respect to $\nu$. This means that $\mu$ is finite. ///

An important corollary of Proposition 3 is that $r$ is a strictly decreasing function of time unless all consumers have the same discount rate.

Corollary 1 For every $t \in \boldsymbol{R}_{+}, r^{\prime}(t) \leq 0$. The weak inequality holds as an equality if and only if $\nu \circ \rho^{-1}$ is concentrated on a single point in $\boldsymbol{R}_{++}$.

Proof of Corollary 1 By Proposition 1,

$$
\frac{d}{d t}\left(-\frac{h^{\prime}(t)}{h(t)}\right) \leq 0
$$

for every $t$, and, by (15), the weak inequality holds as an equality if and only if $\left(\nu \circ \rho^{-1}\right) \circ \beta$ is concentrated on a single point in $\boldsymbol{R}_{++}$. By (6) and the definition of $\beta$, this is equivalent to saying that $r^{\prime}(t)=0$ if and only if $\nu \circ \rho^{-1}$ is concentrated on a single point in $\boldsymbol{R}_{++}$. ///

The following proposition is the main result of this paper, and establishes the converse of Proposition 3. It implies that we can recover the function $\rho$ defining utility weights from $h$. As in the proof of Proposition 3, for a $\beta>0$, we also use $\beta$ to denote the function of multiplying $\beta$, that is, $\beta(q)=\beta q$ for every $q \in \boldsymbol{R}$.

Proposition 4 Let $\nu, \rho$, and $\beta$ be as defined in Section 2. Let $h \in \mathscr{H}$. Let $\mu$ be the unique Borel measure on $\boldsymbol{R}_{++}$that satisfies (8). Then $\mu$ and $\left(\nu \circ \rho^{-1}\right) \circ \beta$ are mutually absolutely continuous if and only if there exists a measurable function $\lambda: A \rightarrow \boldsymbol{R}_{++}$such that $a \mapsto(\lambda(a))^{1 / \beta}$ is integrable with respect to $\nu$ and (5) holds. Moreover, then, there is an essentially unique $\lambda$, with respect to $\mu$ and $\left(\nu \circ \rho^{-1}\right) \circ \beta$, that satisfies (5) and is measurable with respect to the sub- $\sigma$-field generated by $\rho$.

Roughly speaking, this proposition tells us that if we assume that all consumers have equal and constant relative risk aversion and constant but not necessarily equal discount rates, and, in addition, if we know the representative consumer's impatience, then we can fully recover the utility weights given to each discount rate in the welfare maximization problem (1). Put differently, let's assume that all individual consumers have constant and equal relative risk aversion, either because doing so can be justified by some set of data regarding their portfolio choices, or because doing so simplifies the analysis. ${ }^{5}$ By observing the equity premium (the

\footnotetext{
${ }^{5}$ Data hardly justify the assumption of constant and equal relative risk aversion. For example, the survey results of Barsky et. al. (1997) showed that the relative risk aversion is significantly heterogeneous across respondents. The set of panel data of household consumptions in Indian and Pakistani villages of Mazzocco and Saini (2006) showed that the relative risk aversion differ across the households. On the other hand, to test the full risk-sharing hypothesis in Indian and Pakistani villages, Townsend (1994) assumed that all households have constant and equal relative risk aversion, while Ogaki and Zhang (2001) assumed that the first derivatives of the reciprocal of absolute risk aversion is constant and equal across all households. This latter assumption is weaker than the assumption of constant and equal relative risk aversion.
} 
difference between the risk-free interest rate and the expected rate of return on the market portfolio), we can figure out the level $\beta$ of the constant relative risk aversion. By observing the term structure of interest rates, we can figure out the representative consumer's discount factor $d=(d(t))_{t \in \boldsymbol{R}_{+}}$. We can then calculate $h=(h(t))_{t \in \boldsymbol{R}_{+}}$via $h(t)=(d(t))^{1 / \beta}$. Then, by Proposition 4, we can completely recover the utility weight given to each discount rate. Since the utility weights are monotonically related with the wealth shares, this means that once we observe the bond prices, we can recover the wealth distribution among consumers of various discount rates. ${ }^{6}$

Two more remarks on Proposition 4 are in order. First, by claiming that a function $\lambda$ specifying utility weights can take strictly positive values if and only if $\mu$ and $\left(\nu \circ \rho^{-1}\right) \circ \beta$ are mutually absolutely continuous, it shows that the representative consumer's discount factor tells us which discount rates are or are not held by individual consumers in the economy. Second, the proposition only claims the function $\lambda$ specifying utility weight is unique among those which are measurable with respect to the sub- $\sigma$-field generated by the function $\rho$ specifying discount rates. In fact, although we can fully recover utility weights given to each group of consumers of the same discount rate, we can in no way identify how the weights are distributed among consumers in any such group. This is because the mutual fund theorem holds within each such group and the distribution of utility weights within it does not affect the representative consumer's discount factor.

Proof of Proposition 4 Suppose first that there exists a measurable function $\lambda: A \rightarrow \boldsymbol{R}_{++}$ such that $a \mapsto(\lambda(a))^{1 / \beta}$ is integrable and (5) holds. Define $\bar{\lambda}$ and $g$ as in the proof of Proposition 3 , then we obtain (15). This and (8) together imply that the Borel measure $\mu$ coincides with the Borel measure of which (a version of) the Radon-Nikodym derivative with respect to $\left(\nu \circ \rho^{-1}\right) \circ \beta$ is the function $q \mapsto(g(\beta q))^{1 / \beta}$. To establish mutual absolute continuity, it remains to prove that this function takes strictly positive values almost surely with respect to $\left(\nu \circ \rho^{-1}\right) \circ \beta$. Indeed, since $\left(\nu \circ \rho^{-1}\right) \circ \beta=\nu \circ\left(\beta^{-1} \circ \rho\right)^{-1}$, this measure puts probability zero outside the set $\left\{\rho(a) / \beta \in \boldsymbol{R}_{++} \mid a \in A\right\}$. But, for every $q=\rho(a) / \beta$ in this set, $g(\beta q)=\bar{\lambda}(a)>0$ because $\lambda$ takes strictly positive values and $\bar{\lambda}$ is its conditional expectation. Thus the function $q \mapsto(g(\beta q))^{1 / \beta}$ takes strictly positive values almost surely with respect to $\left(\nu \circ \rho^{-1}\right) \circ \beta$.

Suppose conversely that $\mu$ and $\left(\nu \circ \rho^{-1}\right) \circ \beta$ are mutually absolutely continuous. Then the Radon-Nikodym derivative $d \mu /\left(d\left(\left(\nu \circ \rho^{-1}\right) \circ \beta\right)\right)$ takes strictly positive values almost surely. Define $\lambda: A \rightarrow \boldsymbol{R}$ by

$$
\lambda(a)=\left(\frac{d \mu}{d\left(\left(\nu \circ \rho^{-1}\right) \circ \beta\right)}\left(\frac{\rho(a)}{\beta}\right)\right)^{\beta}
$$

for every $a \in A$. Then $\lambda$ takes strictly positive values almost surely with respect to $\nu$, because the Radon-Nikodym derivative takes strictly positive values almost surely on $\left\{\rho(a) / \beta \in \boldsymbol{R}_{++} \mid\right.$

\footnotetext{
${ }^{6}$ If the implied wealth distribution is drastically different from the empirically observed one, we should ponder over how to change our assumptions, especially the assumption of constant and equal relative risk aversion.
} 
$a \in A\}$. Note that

$$
\begin{aligned}
\mu\left(\boldsymbol{R}_{++}\right) & =\int_{\boldsymbol{R}_{++}} \frac{d \mu}{d\left(\nu \circ\left(\beta^{-1} \circ \rho\right)^{-1}\right)}(q) d\left(\nu \circ\left(\beta^{-1} \circ \rho\right)^{-1}\right)(q) \\
& =\int_{A} \frac{d \mu}{d\left(\nu \circ\left(\beta^{-1} \circ \rho\right)^{-1}\right)}\left(\left(\beta^{-1} \circ \rho\right)(a)\right) d \nu(a) \\
& =\int_{A} \frac{d \mu}{d\left(\left(\nu \circ \rho^{-1}\right) \circ \beta\right)}\left(\frac{\rho(a)}{\beta}\right) d \nu(a)
\end{aligned}
$$

Since $\mu\left(\boldsymbol{R}_{++}\right)<\infty$, this shows that $a \mapsto(\lambda(a))^{1 / \beta}$ is integrable. Moreover,

$$
\begin{aligned}
& \int_{A}(\lambda(a))^{1 / \beta} \exp \left(-\frac{\rho(a)}{\beta} t\right) d \nu(a) \\
= & \int_{A} \frac{d \mu}{d\left(\nu \circ\left(\beta^{-1} \circ \rho\right)^{-1}\right)}\left(-\left(\beta^{-1} \circ \rho\right)(a) t\right) \exp \left(-\left(\beta^{-1} \circ \rho\right)(a) t\right) d \nu(a) \\
= & \int_{A} \frac{d \mu}{d\left(\nu \circ\left(\beta^{-1} \circ \rho\right)^{-1}\right)}(-q t) \exp (-q t) d\left(\nu \circ\left(\beta^{-1} \circ \rho\right)^{-1}\right)(q) \\
= & h(t) .
\end{aligned}
$$

To establish the uniqueness of $\lambda$ among those measurable with respect to the sub- $\sigma$-field generated by $\rho$, note from the first paragraph of this proof that since the Radon-Nikodym derivative is essentially unique, the function $q \mapsto(g(\beta q))^{1 / \beta}$ is essentially unique with respect to $\left(\nu \circ \rho^{-1}\right) \circ \beta$, even when $\lambda$ need not be measurable with respect to the sub- $\sigma$-field generated by $\rho$. This means that the function $s \mapsto g(s / \beta)$ is essentially unique with respect to the measure $\nu \circ \rho^{-1}$. Thus the function $a \mapsto g(\rho(a) / \beta)$ is essentially unique with respect to $\nu$. Thus $\bar{\lambda}$ is also essentially unique. This means that there is an essentially unique $\lambda$, up to with respect to $\mu$ and $\left(\nu \circ \rho^{-1}\right) \circ \beta$, that satisfies (5) and is measurable with respect to the sub- $\sigma$-field generated by $\rho$.

Gollier and Zeckhauser (2005, Section IV) showed that if $\nu \circ \rho^{-1}$ is exponentially distributed, then $r$ may be a hyperbolic function. This result shows that hyperbolic discounting may emerge as a consequence of heterogeneous discount rates, even when they are constant for each consumer. The above proposition gives a full characterization of the representative consumer's discount factor even when $\nu \circ \rho^{-1}$ is not exponentially distributed.

\section{Conclusion}

We have proved that if all individual consumers have constant and equal relative risk aversion, and constant but not necessarily equal discount rates, then the representative consumer's discount factor is a power function of some completely monotone function satisfying certain boundary conditions; and that, conversely, any such function can be derived as the representa- 
tive consumer's discount factor from a group of consumers having constant and equal relative risk aversion, and constant but not necessarily equal discount rates. We have also shown that there is a one-to-one correspondence between the distribution of utility weights defining the representative consumer and his discount factor.

While we have obtained a full characterization of the representative consumer's discount factor in the case where all individual consumers have constant and equal relative risk aversion, we have not obtained any characterization of his impatience (discount rates). To do so, we need to find an equivalent condition, in terms of $r$, for $h$ to be completely monotone when $r$ is defined from $h$ via (6). It is important to find such an equivalent condition, because it will tell us which one of the often used models of the term structure of interest rates, presented, for example, in Duffie (2001, Section 7), can be justified by the presence of heterogeneous discount rates.

\section{References}

[1] Robert B. Barsky, F. Thomas Juster, Miles S. Kimball, and Matthew D. Shapiro, 1997, Preference parameters and behavioral heterogeneity: An experimental approach in the health and retirement study, Quarterly Journal of Economics 112, pp. 537-579.

[2] Patrick Billingsley, 1995, Probability and Measure, 3rd ed., John Wiley and Sons, New York.

[3] Darrell Duffie, 2001, Dynamic Asset Pricing Theory, 3rd ed., Princeton University Press, Princeton.

[4] Christian Gollier and Richard Zeckhauser, 2005, Aggregation of heterogeneous time preferences, Journal of Political Economy 113, 878-896.

[5] William Feller, 1966, An Introduction to Probability Theory and its Applications, vol. 2, John Wiley and Sons, New York.

[6] Chiaki Hara, 2006, Heterogeneous risk attitudes in a continuous-time model, Japanese Economic Review 57 (2006), 377-405.

[7] Chiaki Hara, 2007, Heterogeneous impatience in a continuous-time model, working paper, Institute of Economic Research, Kyoto University (Kyoto, Japan).

[8] Chiaki Hara, James Huang, and Christoph Kuzmics, 2007, Efficient risk-sharing rules and the representative consumer's risk aversion, forthcoming in Journal of Economic Theory.

[9] Maurizio Mazzocco and Shiv Saini, 2006, Testing efficient risk sharing with heterogeneous risk preferences: Semi-parametric tests with an application to village economies, manuscript.

[10] Masao Ogaki and Qiang Zhang, 2001, Decreasing relative risk aversion and tests of risk sharing, Econometrica 69, 515-526.

[11] Robert Townsend, 1994, Risk and insurance in village India, Econometrica 62, 539-591. 